\title{
Terahertz aperture SNOM mapping of metamaterial coupled resonators
}

\author{
Almond, Nikita, Hermans, Rodolfo, Hale, Lucy, Kindness, \\ Stephen, Michailow, Wladislaw, et al.
}

Nikita W. Almond, Rodolfo Hermans, Lucy L. Hale, Stephen J. Kindness, Wladislaw Michailow, Binbin Wei, Xavier Romain, Sheng Ye, Robert Young, Riccardo Degl'Innocenti, Harvey E. Beere, David A. Ritchie, Oleg Mitrofanov, "Terahertz aperture SNOM mapping of metamaterial coupled resonators," Proc. SPIE 11460, Metamaterials, Metadevices, and Metasystems 2020, 114601R (20 August 2020); doi: 10.1117/12.2568045

SPIE. Event: SPIE Nanoscience + Engineering, 2020, Online Only 


\title{
Terahertz Aperture SNOM Mapping of Metamaterial Coupled Resonators
}

\author{
Nikita W. Almond ${ }^{\mathrm{a}}$, Rodolfo Hermans ${ }^{\mathrm{b}}$, Lucy L. Hale ${ }^{\mathrm{b}}$, Stephen J. Kindness ${ }^{\mathrm{a}}$, Wladislaw \\ Michailow $^{\mathrm{a}}$, Binbin $\mathrm{Wei}^{\mathrm{a}}$, Xavier Romain ${ }^{\mathrm{c}}$, Sheng Ye ${ }^{\mathrm{c}}$, Robert Young ${ }^{\mathrm{c}}$, Riccardo \\ Degl'Innocenti ${ }^{\mathrm{c}}$, Harvey E. Beere ${ }^{\mathrm{a}}$, David A. Ritchie ${ }^{\mathrm{a}}$, and Oleg Mitrofanov ${ }^{\mathrm{b}}$ \\ ${ }^{a}$ Cavendish Laboratory,University of Cambridge, J J Thomson Avenue, Cambridge, CB3 0HE, \\ UK \\ ${ }^{\mathrm{b}}$ University College London, Electronic and Electrical Engineering, London, WC1E 7JE, UK \\ ${ }^{\mathrm{c}}$ Department of Engineering, University of Lancaster, Bailrigg, Lancaster, LA1 4YW, UK
}

\begin{abstract}
Metamaterials have emerged as the basis of a novel optoelectronic platform operating in the terahertz ( $\mathrm{THz})$ range, due to their versatility and strong light-matter interaction. The necessary design of efficient modulators and detectors requires a detailed investigation of metamaterial resonances and their interplay with an active medium, e.g. graphene. An aperture-SNOM (a-SNOM) system based on picosecond $\mathrm{THz}$ pulses was used to investigate the spectral characteristics of a set of lithographically tuned metamaterial coupled resonators. This approach allowed the mapping of the supported E-field of each resonator a few microns from the device plane, yielding bonding and antibonding modes reminiscent of electromagnetic induced transparency.
\end{abstract}

Keywords: Metasurface, metamaterial, EIT, a-SNOM, near-field, Terahertz, TDS

\section{INTRODUCTION}

Metamaterials provide a method of manipulating terahertz $(\mathrm{THz})$ radiation that is not achievable with naturally occurring materials. Metamaterials are formed of sub-wavelength resonators forming artificial atoms. It is possible using metamaterials to design the dispersive response of the material paving the way for a multitude of $\mathrm{THz}$ components, such as amplitude/phase modulators, as well as chiral and beam steering devices or spatial light modulators. ${ }^{1-3}$ These elements could enable essential functionalities such as OOK (on-off key) or QAM (quadrature amplitude modulation) and polarisation shift keying for communications, ${ }^{4}$ spectroscopy for pharmaceuticals and microscopy for semiconductor device analysis.

The investigation of the terahertz optical response of metamaterial resonant devices normally requires the engineering of arrays composed of identical unit cells, to increase light coupling. However, now with the growing field of $\mathrm{THz}$ near-field microscopy, it is possible to optically address a single resonator without the need of fabricating large arrays. Scattering-SNOM could be used to explore individual resonators, as has been done in Ref. 5. Another such technique is aperture- scanning near-field optical microscopy (a-SNOM) and this is the technique used here. This combines $\mathrm{THz}$ time-domain spectroscopy (TDS) with a sub-wavelength aperture integrated with a photoconductive antenna detector, placed in close proximity to the sample surface. The setup is described in more detail in Ref. 6 and has been used to explore metamaterials in Ref. 7. The coupled resonators imaged here are lithographic variations of the device reported in Ref. 8,9 which consists of an array of identical metamaterial unit cells exhibiting optical features reminiscent of the electromagnetic induced transparency in atomic physics.

Further author information: (Send correspondence to N.W.A.)

N.W.A.: E-mail: na470@cam.ac.uk 

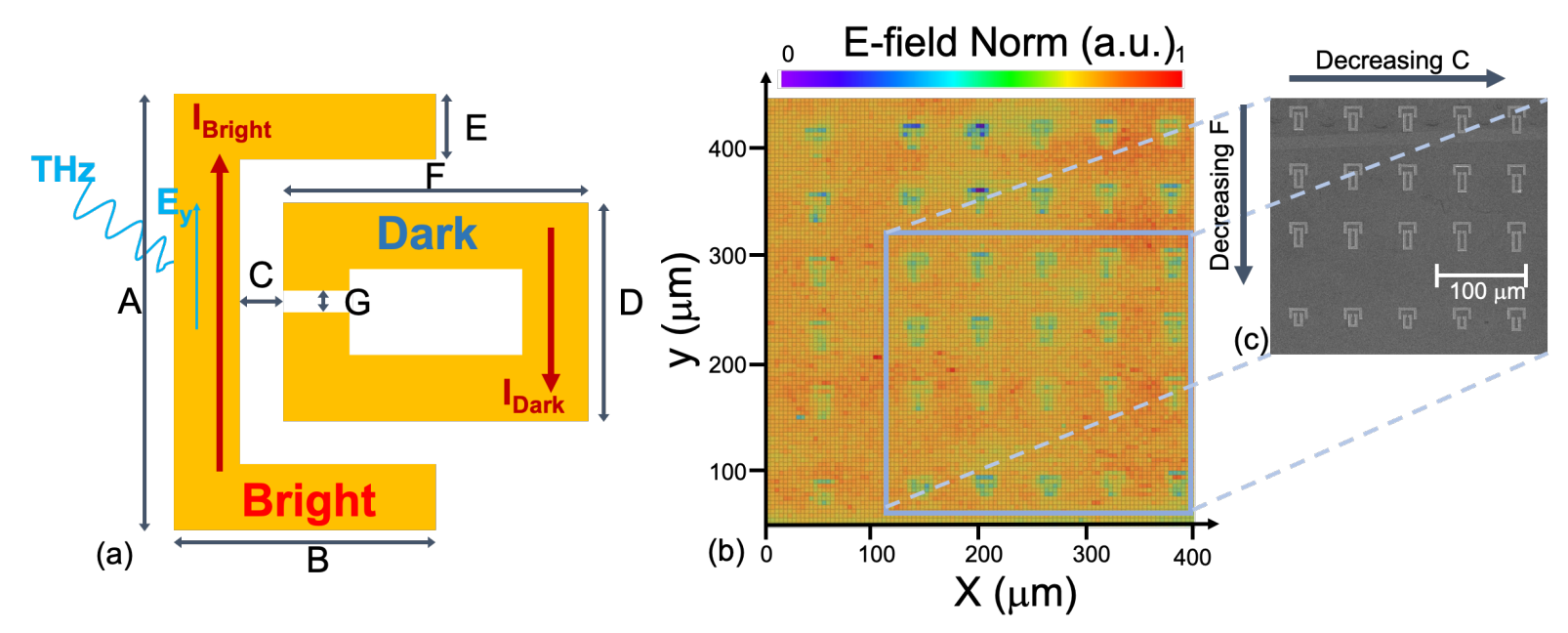

Figure 1. (a) Schematic of coupled resonators, the current indicated is for the bonding mode, (b) is an xy field map taken with the a-SNOM at a fixed time after the THz pulse and (c) inset is a SEM image of the resonators.

\begin{tabular}{|c|c|}
\hline Parameter & Value $(\boldsymbol{\mu m})$ \\
\hline $\mathrm{A}$ & 20 \\
\hline $\mathrm{B}$ & 12 \\
\hline $\mathrm{C}$ & $2-7$ \\
\hline $\mathrm{D}$ & 10 \\
\hline $\mathrm{E}$ & 3 \\
\hline $\mathrm{F}$ & $14-21$ \\
\hline $\mathrm{G}$ & 1 \\
\hline
\end{tabular}

Table 1. table of the geometric parameter values for the resonators.

\section{FABRICATION}

The sample was fabricated using standard cleanroom lithographic techniques. A p-doped silicon substrate with $300 \mathrm{~nm}$ of silicon dioxide was used as a substrate, to allow all-electrical tuning of the resonances via back-gating. Electron beam lithography was used to define the individual resonators, which were formed of 10/70 $\mathrm{nm}$ of $\mathrm{Ti} / \mathrm{Au}$ which was deposited via thermal evaporation. A schematic of the resonators is shown in figure 1a and a scanning electron microscopy (SEM) image of an array of individual distinct coupled resonators is shown in figure 1c. Moving down the sample, ' $F$ ' decreases, this changes the resonant frequency of the dark resonator. Moving across the sample decreases ' $\mathrm{C}$ ', this separates the resonators and reduces the coupling between the bright and dark resonator, giving rise to sharper features for the bonding resonance. The parameters used to define the dimensions of the resonators and the ranges of them are shown in table 1 . There is a $60 \mu \mathrm{m}$ pitch in both $\mathrm{x}$ and $y$ between the resonators which is large enough to prevent adjacent elements from interacting strongly with each other and affecting their resonant frequencies, although they will likely still be some scattered field from adjacent resonators present.

\section{SIMULATIONS}

The metamaterial resonators were simulated using finite element modelling (FEM), COMSOL Multiphysics 5.5, RF package, and the Drude model was used to describe the complex conductivity of the gold. The array was simulated using a single unit cell with periodic Floquet boundary conditions. A port was used to simulate the incoming radiation, while the S11 and S21 parameters were recorded to calculate the reflection and the transmission of the device, respectively. 

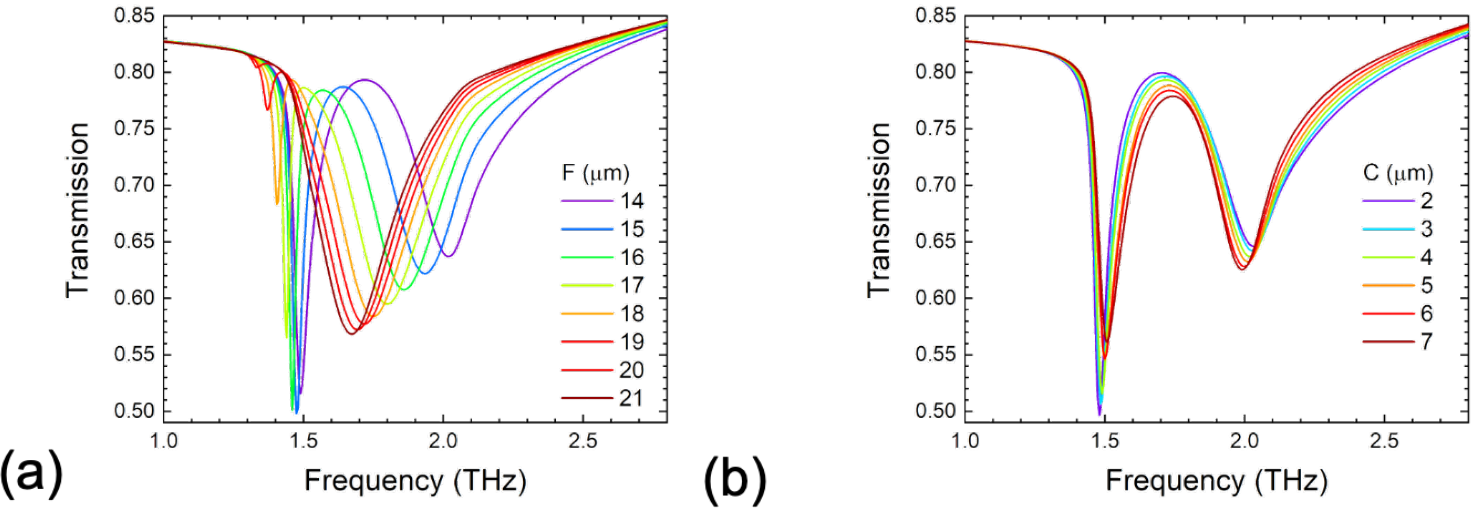

Figure 2. Comsol simulations showing the far-field S21 parameter for an array of identical coupled resonators as a function of (a) 'F' with C fixed at $4 \mu \mathrm{m}$, and (b) 'C' with F fixed at $14 \mu \mathrm{m}$.

As in Ref. 9 each coupled-resonator is formed of two C-shaped resonators whose resonant frequency is close together, within a few $100 \mathrm{GHz}$. The resonators form a bright and a dark resonator, shown in figure 1a. The larger C-shaped resonator is directly excited by y-polarised incoming THz radiation. The resonance is centred at $1.54 \mathrm{THz}$. Due to the capacitive coupling between the two resonators, the smaller dark C-shaped resonator is excited. The smaller resonator is 'dark' as it is subradient due to the short component in the y-direction that is parallel to the incoming terahertz radiation that leads it to only weakly interact with this field. It is excited by the strong near-field capacitive coupling with the 'bright' resonator due to the long element in the y-direction. The two resonators each support localised surface plasmons (LSP) at similar frequencies. These C-shaped elements supporting similar resonant frequencies appear to split due to the near-field coupling into two hybrid modes, a bonding and an anti-bonding mode. The coupled resonators can be described by an electrically induced transparency analogue.

The simulated far-field emission from an array of identical resonators with varying ' $\mathrm{F}$ ' and ' $\mathrm{C}$ ' parameters are shown in figures $2 \mathrm{a}$ and $2 \mathrm{~b}$ respectively. As ' $\mathrm{F}$ ' increases the frequency of the LSP decreases, this brings the resonances of the bright and dark resonators closer together and leads to a stronger coupling. As the width of the dark C-shaped resonator increases towards the extreme of $21 \mu \mathrm{m}$, the sharp dip from the anti-bonding mode reduces as the resonances are too close. As ' $\mathrm{C}$ ' increases, the coupling between the resonators reduces as the near-field reduces rapidly with increasing distance.

Figures 3a\&b show the $\mathrm{z}$ component of the electric field $10 \mathrm{~nm}$ above the metal surface of the resonators. It shows the case when ' $\mathrm{C}$ ' $=4 \mu \mathrm{m}$ and $\mathrm{F}=16 \mu \mathrm{m}$. These are the dimensions for the device measurements presented here. The bonding mode can be seen in figure $3 \mathrm{a}$, the currents in both resonators are in the same direction. The anti-bonding modes can be seen in figure 3b, where the currents are now in opposite directions.

\section{RESULTS: APERTURE SNOM}

An a-SNOM described in Ref. 6 was used to perform XY maps, measuring the spatial distribution of the field at a fixed point in time after the pulse, and XT maps, measuring the time evolution of the field for each point along a line, of individual resonators on the sample. An infrared laser and a ZnTe crystal were used to generate the THz pulses. A $10 \mu \mathrm{m}$ aperture was used to probe the near-field close to the surface of the resonators. The aperture is integrated with a photoconductive antenna which was used for detection.

The sample was excited along the y-direction as in the simulations. The $\mathrm{THz}$ field launches surface waves that are dependent on the resonance of the resonators. This setup allows the time evolution of the electric field to be probed for sub-wavelength areas on the resonators. In this way a near-field map can be created in $\mathrm{x}, \mathrm{y}$ and $\mathrm{t}$, allowing the response of individual resonators to the $\mathrm{THz}$ field to be found. 

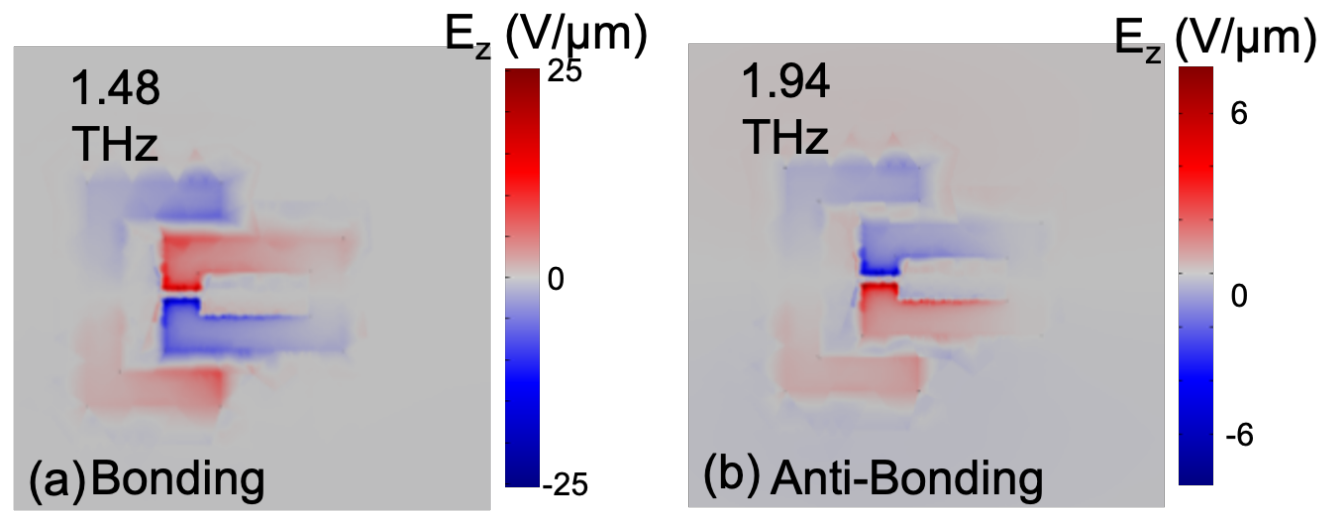

Figure 3. The simulated $\mathrm{E}_{z} 10 \mathrm{~nm}$ above the gold resonator surface for $\mathrm{C}=4 \mu \mathrm{m}$ and $\mathrm{F}=16 \mu \mathrm{m}$ at (a) $1.48 \mathrm{THz}$ and (b) $1.94 \mathrm{THz}$

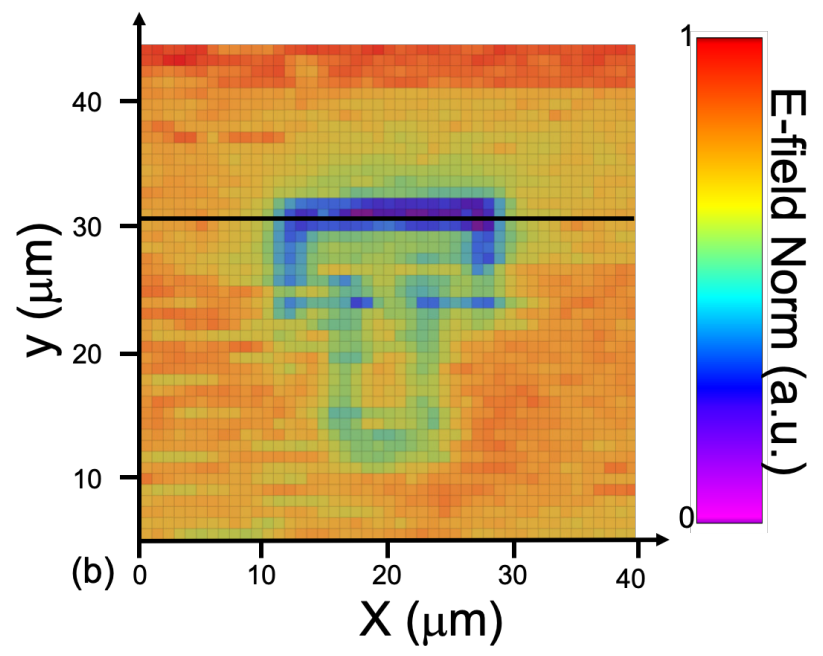

Figure 4. $\mathrm{XY}$ field scan of coupled resonators, $\mathrm{C}=4 \mu \mathrm{m}$ and $\mathrm{F}=16 \mu \mathrm{m}$, delay of 1.4 ps after the peak.

With the delay set to probe only a few picoseconds after the pulse, $1.4 \mathrm{ps}$ after the peak, at $2.7 \mathrm{ps}$ on the $\mathrm{XT}$ scans, the sample was raster scanned in $\mathrm{x}$ and $\mathrm{y}$ to make a map, thus achieving the XY scan shown in figure 4. The bright resonator shows a much larger field than the dark resonator and above that expected from simply a contrast between gold and silicon. This is as expected as the bright resonator will interact with the incident $\mathrm{THz}$ radiation more strongly.

Time-domain scans were performed across the line shown in Figure 4, where the greatest contrast was expected. At each spatial position, the time delay was varied to build up images showing the field evolution in time for the line. This is shown in figure 5a. Periodic surface waves are clearly visible along the line and there is a clear contrast between the gold and silicon. In fig. 4a, a single surface wave is seen, indicating only one excited resonance. The spatial position matches the large metallic part of the larger C-shaped resonator parallel to the incident field, at 10-30 $\mu \mathrm{m}$ in figure 4, this corresponds to the bright resonator. The approximate period of this wave is 0.68 ps corresponding to a frequency of $1.46 \mathrm{THz}$, which matches the dip in figure 2a corresponding to the bonding mode.

After doing a fast Fourier transform (FFT) at each spatial point in figure 5a, and normalising the data to the incident waveform, figure $5 \mathrm{c}$ was produced. The acquisition is relatively noisy, with this effect being attributed to the doped substrate. However, it still confirms what the XT scan shows (Fig. 5a) that there is one LSP present 

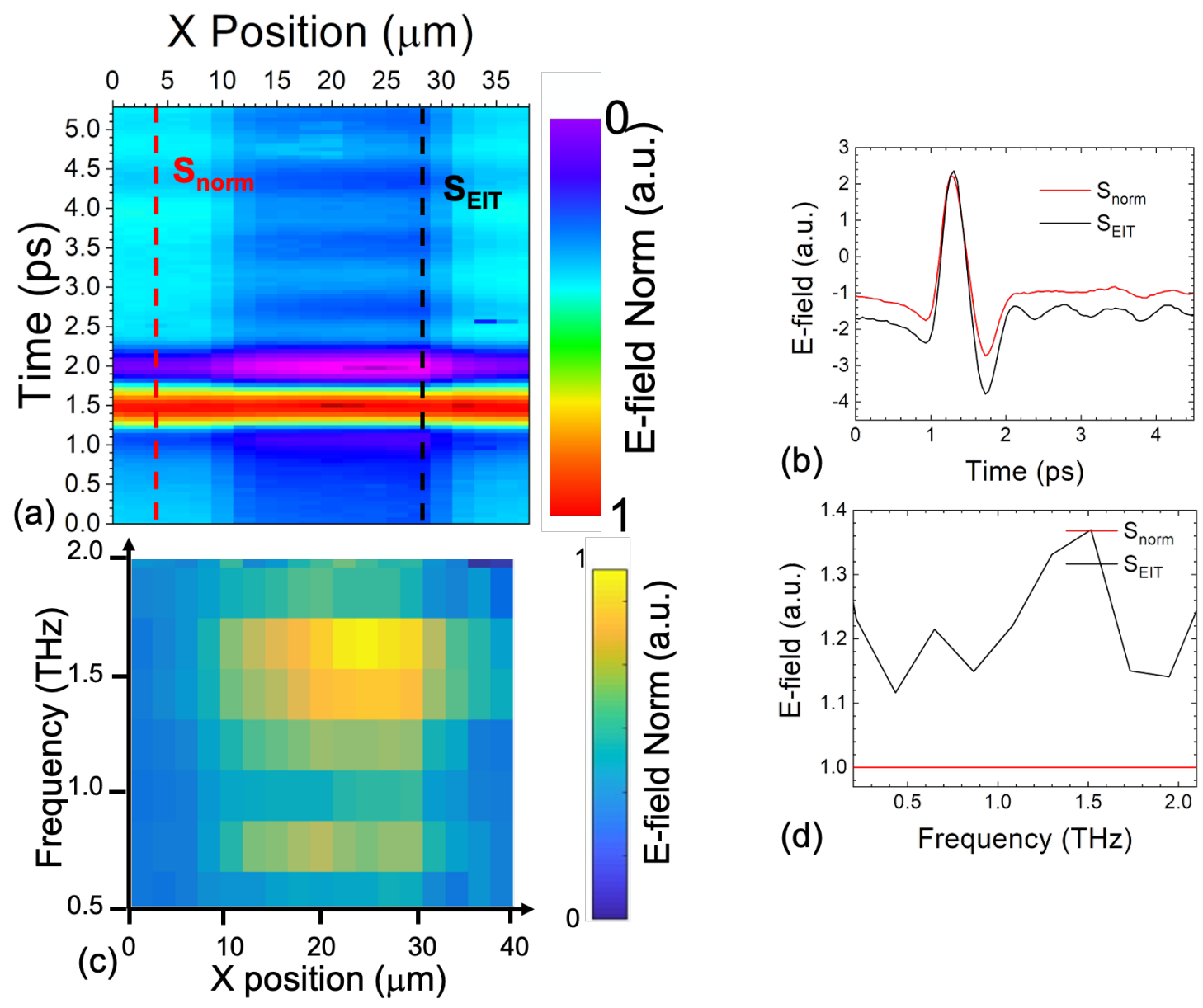

Figure 5. (a) XT scan along the line parallel to the bright resonanor bar at $\mathrm{y}=32 \mu \mathrm{m}$, (b) temporal waveforms extracted along the indicated lines. The time scans were Fourier transformed to produce (c) and line scans at the same position are shown in $(d)$. 
in the line scan across the line with a frequency of $\sim 1.5 \mathrm{THz}$, which agrees with the simulations, although a better resolution is needed for greater accuracy.

Further investigations are needed to better explore the near-field coupling between the coupled resonators as the parameters are varied to assist with device design for applications of THz metasurfaces. As with the devices reported in Ref. 9 graphene was added to some features, not shown here, so further imaging will be undertaken with varying back-gate voltages.

\section{ACKNOWLEDGMENTS}

The authors acknowledge EPSRC funding within the Hyperterahertz grant, number EP/P021859/1, Jonathan P. Griffiths and Thomas A. Mitchell for help with electron beam lithography. R.D. acknowledges support from the EPSRC (Grant No EP/S019383/1).

\section{REFERENCES}

[1] Li, Y., Tantiwanichapan, K., Swan, A. K., and Paiella, R., "Graphene plasmonic devices for terahertz optoelectronics," Nanophotonics 9(7), 1901 - 1920 (2020).

[2] Low, T. and Avouris, P., "Graphene plasmonics for terahertz to mid-infrared applications," ACS Nano 8(2), 1086-1101 (2014). PMID: 24484181.

[3] Shi, J., Li, Z., Sang, D. K., Xiang, Y., Li, J., Zhang, S., and Zhang, H., "Thz photonics in two dimensional materials and metamaterials: properties, devices and prospects," J. Mater. Chem. C 6, 1291-1306 (2018).

[4] Dhillon, S. S., Vitiello, M. S., Linfield, E. H., Davies, A. G., Hoffmann, M. C., Booske, J., Paoloni, C., Gensch, M., Weightman, P., Williams, G. P., Castro-Camus, E., Cumming, D. R. S., Simoens, F., EscorciaCarranza, I., Grant, J., Lucyszyn, S., Kuwata-Gonokami, M., Konishi, K., Koch, M., Schmuttenmaer, C. A., Cocker, T. L., Huber, R., Markelz, A. G., Taylor, Z. D., Wallace, V. P., Zeitler, J. A., Sibik, J., Korter, T. M., Ellison, B., Rea, S., Goldsmith, P., Cooper, K. B., Appleby, R., Pardo, D., Huggard, P. G., Krozer, V., Shams, H., Fice, M., Renaud, C., Seeds, A., Stöhr, A., Naftaly, M., Ridler, N., Clarke, R., Cunningham, J. E., and Johnston, M. B., "The 2017 terahertz science and technology roadmap," Journal of Physics D: Applied Physics 50, 043001 (Jan 2017).

[5] Degl'Innocenti, R., Wallis, R., Wei, B., Xiao, L., Kindness, S. J., Mitrofanov, O., Braeuninger-Weimer, P., Hofmann, S., Beere, H. E., and Ritchie, D. A., "Terahertz nanoscopy of plasmonic resonances with a quantum cascade laser," ACS Photonics 4(9), 2150-2157 (2017).

[6] Mitrofanov, O., Lee, M., Hsu, J. W. P., Brener, I., Harel, R., Federici, J. F., Wynn, J. D., Pfeiffer, L. N., and West, K. W., "Collection-mode near-field imaging with 0.5-thz pulses," IEEE Journal of Selected Topics in Quantum Electronics 7(4), 600-607 (2001).

[7] Hale, L. L., Keller, J., Siday, T., Hermans, R. I., Haase, J., Reno, J. L., Brener, I., Scalari, G., Faist, J., and Mitrofanov, O., "Noninvasive near-field spectroscopy of single subwavelength complementary resonators," Laser 85 Photonics Reviews 14(4), 1900254 (2020).

[8] Chen, C.-Y., Un, I.-W., Tai, N.-H., and Yen, T.-J., "Asymmetric coupling between subradiant and superradiant plasmonic resonances and its enhanced sensing performance," Opt. Express 17, 15372-15380 (Aug ts , url = http://www.opticsexpress.org/abstract.cfm?URI=oe-17-17-15372, doi = 10.1364/OE.17.015372,).

[9] Kindness, S. J., Almond, N. W., Wei, B., Wallis, R., Michailow, W., Kamboj, V. S., Braeuninger-Weimer, P., Hofmann, S., Beere, H. E., Ritchie, D. A., and Degl'Innocenti, R., "Active control of electromagnetically induced transparency in a terahertz metamaterial array with graphene for continuous resonance frequency tuning," Advanced Optical Materials 6(21), 1800570 (2018). 